\title{
Leach Protocol in Wireless Sensor Networks using Matlab
}

\author{
Parneet kaur, Pooja Sahni, Sukhdeep Kaur
}

\begin{abstract}
Today, at this age of intelligent technology, the intelligent have entered agriculture in a great way. The base of this study is WSN based irrigation system. In this work various problems in the irrigation system based on traditional wireless sensor networks. The flaw is related to the selection approach for $\mathrm{CH}$ (cluster head). The most important technologies are used in this work i.e. to develop an automatic and intelligent irrigation system. The use of a sensor monitoring system is an effective method to prevent interference and to improve efficiency. The $\mathrm{CH}$ election approach is modified by enhancing the list of network influencing factors. To sense the energy of the overall network, LEACH and IMPROVED LEACH protocol are compared to have a better and efficient network. MATLAB simulates and evaluates the performance of the communication system in terms of network life. The results of the simulation show the different amount of sensors initial energy in relation to the dead last node and the dead first node.
\end{abstract}

Index Terms: WSN (wireless sensor networks), cluster head, MATLAB

\section{INTRODUCTION}

The Wireless Sensor Network basically contains the gateway nodes and a large number of independent sensor nodes. Due to small size, sensor nodes are also named as Motes. They use attached sensors for calculating and collecting environmental information like light condition, pressure, humidity, and temperature etc. The information reaches the gateway through a network with the help of mote. During the forwarding, different routes might be taken depending on

the availability of the sensor nodes routing tables. The gateway node is normally connected to a computer from a different network in

order to gather all the data sent from the WSN. The combination of computing and communication, distributed sensing forms a wireless sensor network [17]. These Wireless Sensors are ad-hoc networks, where each sensor node participates in routing by forwarding information to other sensor nodes using the nearest neighboring communication. Without the use of a pre-defined infrastructure until the gateway is reached These gateways might be bridged with other gateways either to combine networks such as the

Revised Manuscript Received on July 09, 2019.

Parneet Kaur, Department of Electronics and Communication Engineering, Chandigarh Engineering College, Landran, Mohali, India

Dr. Pooja Singh, Department of Electronics and Communication Engineering, Chandigarh Engineering College, Landran, Mohali, India

Dr. Sukhdeep Kaur, Department of Electronics and Communication Engineering, Chandigarh Engineering College, Landran, Mohali, India
Internet or to extend the data transmission to a location where it is difficult for sensor nodes to arrive.

Table 1. Comparison of Traditional Networks and Wireless Sensor Networks

\begin{tabular}{|l|l|}
\hline Traditional Networks & Wireless Sensor Networks \\
\hline $\begin{array}{l}\text { General purpose design; } \\
\text { serving many applications }\end{array}$ & $\begin{array}{l}\text { Single purpose design; serving the } \\
\text { specific one application }\end{array}$ \\
\hline $\begin{array}{l}\text { Typical Primary design } \\
\text { concerns are network } \\
\text { performance and latencies; } \\
\text { energy is not a primary } \\
\text { concern }\end{array}$ & $\begin{array}{l}\text { Energy is the main constraint in the } \\
\text { design of all node and network } \\
\text { components }\end{array}$ \\
\hline $\begin{array}{l}\text { Networks are designed and } \\
\text { Engineered according to } \\
\text { according to plans }\end{array}$ & $\begin{array}{l}\text { Deployment, Network structure, } \\
\text { and resource use are often ad hoc } \\
\text { (without planning) }\end{array}$ \\
\hline $\begin{array}{l}\text { Devices and Networks are } \\
\text { operate in controlled and } \\
\text { mild environments }\end{array}$ & $\begin{array}{l}\text { Sensor networks often operate in } \\
\text { environments with harsh } \\
\text { conditions }\end{array}$ \\
\hline $\begin{array}{l}\text { Maintenance and Repair are } \\
\text { common and networks are } \\
\text { typically easy to access }\end{array}$ & $\begin{array}{l}\text { Physical access to sensor nodes is } \\
\text { often difficult or even impossible }\end{array}$ \\
\hline $\begin{array}{l}\text { Component Failure is } \\
\text { addressed through } \\
\text { maintenance and repair }\end{array}$ & $\begin{array}{l}\text { Component Failure is expected } \\
\text { and addressed in the design of the } \\
\text { network }\end{array}$ \\
\hline $\begin{array}{l}\text { Obtaining global network } \\
\text { knowledge is typically } \\
\text { feasible and centralized } \\
\text { management is possible }\end{array}$ & $\begin{array}{l}\text { Most decisions are made localized } \\
\text { without the support of a central } \\
\text { manager. }\end{array}$ \\
\hline
\end{tabular}

\section{RELATED WORK}

I Shunmin Wang, [1], (2014), Using 4G innovation for spatial data mining, inspected in context of the high precision water framework feathery spatial data mining and vigilant essential initiative drew in with issues related to a sharp decision candidly steady system is used in this paper. [Method] Set forward by joining cushy clustering examination methodology for the transitive end procedure and modernized picture taking care of in the eight related through calendar estimation technique, so as to recognize precise water framework spatial soft gathering. Remembering the true objective to deal with the issue of the water framework with high precision, put forth the relating cushy batching high dimensional spatial data and streamlining algorithm.[Results] The end through the examination, on the, begin of ensuring 
provincial water framework, water framework structure progression system can comprehend saving over $20 \%$ of water framework water use, improving water framework structure is incredibly necessary.[Conclusions] By using spatial gathering new water system innovation, for instance, high precision for precise water system on spatial fluffy bunching investigation, and put forth broad gauges about high exactness accuracy of water framework and the procedure, along these lines achieving high precision water framework application and spread.

II Jin Wei and Gihan J. Mendis, [2], (2016), the observing quality and savvy matrix's controls were improved by processing applications and interchanges knowledge. Though the odds of malignant assaults like false information infusion expanded on its reliance on data innovation. So as to ease and recognize the data debasement in the issue produced, a learning-based digital physical convention was exhibited in this paper. The introduced system realizes the profound learning strategy to analyze the constant estimation data from the land disseminated Phasor Estimation Units (PMUs) and utilizes the physical knowledge in the power structures to test and perceive the data contamination. We show the execution of the proposed procedure through the diversion by using the New Britain 39-transport control framework.

III Peng Zhang, Qian Zhang, Fusheng Liu, Changqing Song, [3], (2017), With the coming of developing innovations, for example, the IOT(Internet of things) and huge information, the pace of change from customary horticulture to present day agribusiness will be quickened. Given that customary farming exists numerous issues as of now, for example, low usage of water system water and in reverse in the board level, the incorporation of water and manure water system astute enormous information framework is set up dependent on the advances of the Web of things, huge information, etc. The framework utilizes the IOT and some different advances to genuine auspicious screen and consequently gathers the information that is connected with the development of every single yield in the fields and after that transfer them to Shandong Horticultural College huge information focal target database. The focal point of huge information keenly stores, screens, aligns, mines and concentrates the observing information to set up the yield development display dependent on huge information, which can foresee and gauge necessity of water in harvests in different development periods and settle on the choice of programmed water system and preparation, at long last acknowledge auspicious and appropriate water system of harvests.

IV Sandeep Kaur, Deepali, [4], (2017), in nowadays WSN is extensively used as a piece of structure a choice emotionally supportive network for watching and controlling different ecological parameter. A standout amongst the most intriguing fields having an extending requirement for the choice emotionally supportive network is horticulture. The prevalence of WSN in horticulture is because of a few reasons like elite, expanding the generation while diminishing cost, low-control utilization and gathered dispersed information. In this paper, the proposed Mechanized Water system Framework (AIS) is helpful to detect temperature, daylight, moistness, $\mathrm{pH}$, and groundwater in the field and take the choice of necessities of water based on its outcomes.
V Manpreet Kaur, Amarvir Singh, [5], (2016), WSN is a developing innovation due to its immense scope of utilization out in the open and military region. These sensor systems comprising of thousands of little sensor hubs with restricted assets with a base station and ease, low power sensor hubs that are utilized for the checking reason. As these sensor hubs are little in size and substantial in number, subsequently, these systems are effectively inclined to assault. There can be numerous conceivable assaults on sensor arrange like sink-gap assault, dark opening assault, DOS assault, sticking. Among the majority of the above assaults, Sink-gap assault is viewed as most harming steering assault. The issue identified with the sinkhole assault is the subverted hub or the vindictive hub promotes the alluring steering data and powers the hubs to course the information towards it and makes an effective reach. In this manner, sink-gap assault is the sort of assault which debases the execution of the system. The point of this paper is to break down and distinguish the sinkhole assault in the remote sensor organize and to propose the system to expel this assault to verify the system.

VI Ravi Kishore Kodali, Borade Samar Sarjerao, [4], (2017),

Horticulture assumes an imperative job in India. In India, a guess of 50 percent of the all-out populace, straightforwardly or in a roundabout way depended on agribusiness related exercises that to a great extent influence the Indian economy. A rancher needs to confront a ton of issues like checking the water level in the field or to turn the water siphon on or off, now and then even amidst the night. Old techniques for cultivating were should have been improved, so as to beat this issue. Another framework was should have been structured that aided in changing over the old conventional cultivating into keen cultivating. A straightforward water siphon controller was planned with the assistance of soil dampness and Esp8266 NodeMCU-12E. For sending and getting of a sign, a message Line Telemetry Transport convention was utilized. NodeMCU-12E was utilized for controlling activities of the water siphon and for showing the status of sensor information and water siphon on a versatile application to the page. So for tackling the issues referenced over, an adaptable, secure, affordable, and trustable framework was produced.

VII Mahammad Shareef Mekala, Dr P. Viswanathan, [5], (2017), In India, IoT for example Web of Things is viewed as one of the quickest creating innovation. Though $70 \%$ of the populace in India relies on farming. So this circumstance goes about as a principle reason in the postponing of the nation's improvement. So to beat this issue, shrewd farming was should have been exhibited instead of customary agribusiness strategies. So another IoT based innovation was given the assistance of $\mathrm{Li}-\mathrm{Fi}$ and distributed computing. The term Wi-Fi was useful for just broad remote inclusion inside the structure while the term $\mathrm{Li}$-Fi was utilized for remote information inclusion inside extraordinary thickness in limited regions. Li-Fi has effectively obtained seriously rapid in the lab and it additionally gave extraordinary proficiency, accessibility, security, and transmission capacity when contrasted with that of Wi-Fi. Right off the bat the remote-controlled procedures like winged animal and creature frightening, weeding, dampness detecting, keeping recognition and splashing, and so forth were incorporate into this 
undertaking. Besides, shrewd stockroom the executives that contained robbery identification, mugginess, and temperature support was likewise a piece of it. Thirdly for savvy water system with brilliant control, insightful basic leadership based on accurate continuous field data are likewise incorporated into it. The majority of the activities depicted above were controlled will any remote brilliant gadget associated with the Web and every one of the tasks were performed with the assistance of $\mathrm{Li}-\mathrm{Fi}$, sensors, ZigBee module or interfacing cameras.

\section{CLUSTERING}

In the past few years, the use of WSNs has been increased and simultaneously the problems related to the energy constrained also raised because we have a limited amount of battery's energy. Replacement and recharging of battery is not an easy task because the working of nodes totally depends upon the amount of energy. If any node in the network stops operating then it leads to failure of the whole network. To preserve the energy during the communication of data, the following steps are implemented [1].

- Scheduling sensor are the nodes state (i.e. idle, transmitting, sleep or receiving).

- The Implementing appropriate clustering paradigm for network creation.

- By implementing the optimum of routing techniques. The minimum number of sleeping nodes should be activated in order to reduce energy consumption. All the sensor nodes can be in any state either active, idle and sleep while communication of data.

Active mode: In this mode, energy is consumed while receiving and transmitting of information.

Idle mode: The rate of energy consumption is similar to that of the active mode.

Sleep mode: In this mode, radios of sensor nodes are closed to save energy and therefore it can be declared that this mode is totally energy saving the mode.

\section{PROPOSED WORK}

After having a review in the previous work [4], it has been concluded that it suffers from various issues as the scheme of cluster head selection was not quite effective and the system stores the data on the dedicated node i.e. sink node which makes it difficult to access by sitting far away. As we know, the Indian climate is so unpredictable, thus for farmers, it becomes difficult to visit the fields during the poor climatic conditions. Thus, in the present system, a novel approach is developed in which the major technology is WSN.

In this work, the $\mathrm{CH}$ (Cluster Head) selection scheme is also updated by enhancing the count of parameters. The factors considered are the energy of the nodes, the distance from a node to sink node and distance of the node from its adjacent nodes. Along with this, the weight value is evaluated as follows:

$$
\begin{aligned}
& 1=w_{1}+w_{2}+w_{1} \ldots \ldots(4.1) \\
& 1=e_{0}+d_{n}+d_{g} \ldots . .(4.2) \\
& 1=0.6+0.2+0.2 \ldots \ldots .(4.3)
\end{aligned}
$$

Where $w_{1}$ defines the energy, $w_{2}$ defines the node to node distance and $w_{2}$ defines the sink to node distance. In this work, the high weight is given to the energy of the nodes and this is done to increase the accuracy and the lifetime of the network.

\section{RESULTS AND DISCUSSION}

MATLAB is a processing domain alongside an abnormal state, translated, powerfully composed language, supporting practical, object-situated, and occasion coordinated models. It is significant for math calculations, explicitly calculations involving lattice applications and straight variable based math. Fantastic help has been given by MATLAB to information representation and its brief and expressive language structure, just as the plenty of predefined capacities that outcome in a dynamic situation phenomenal for quick prototyping with insignificant overhead. Not with standing, MATLAB isn't only a scripting language for quick and messy calculations. Most recent forms have inspected a power increment for the help of a substantial scale, very organized code to equal the dialects like $\mathrm{C}++$, Java and numerous others. In the event that you need the best of the two universes, MATLAB'S coordinated java bolster gives you a chance to make and control cases of java classes directly in your MATLAB programs. We can likewise shout to the code written in $\mathrm{C}, \mathrm{C}++$, Perl, FORTRAN, or execute dos or UNIX framework directions. Additionally, the MATLAB code can be transported for use in Java, C, C++, and. Net projects, or as an independent task, mitigating its prime hardship - that it isn't free. As a translated language, a few activities are slower when contrasted with ordered language, especially those including circles. This also has improved in late forms. Circles can be vectorized anyway much of the time, bringing about extremely quick code that interests to the low dimension, exceptionally progressed, arranged activities from the MATLAB library.

At the point when the most computationally imperative segments of your program can be composed along these lines, the spryness of MATLAB code matches that of arranged dialects. At long last, with the expansion of different toolboxes, for example for Insights, Bioinformatics, Improvement, or Picture Preparing and so on, the MATLAB programming can be reached out for progressively concentrated necessities.

\section{A. EXPERIMENTAL ANALYSIS:-}

The graph in figure 1 and 3 shows the lifetime of the network in terms of the first node dead and last node dead. The performance of the work is evaluated in terms of network lifetime. The network lifetime is measured with respect to the first dead and last dead node in the network. Here the evaluation is done on the basis of the energy assigned to the nodes. In this case, the energy varies from 0.1 joule to 0.9 joule. The graph in figure 1 depicts the lifetime of the network with respect to the first node dead. The graph proves that the highest energy at nodes enhances the performance and network lifetime. As shown in the graph, in case of highest energy, the first dead node found at later stage. Similarly, the graph in figure 3 provides a graphical representation to the last node dead in similar manner. 


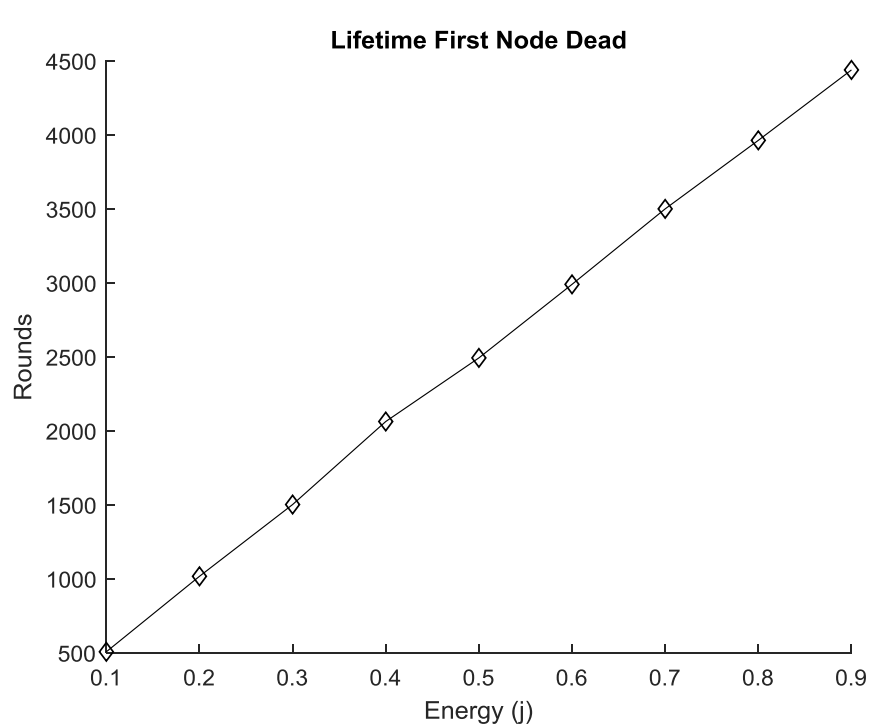

Figure 1: First node dead with respect to the different energy level

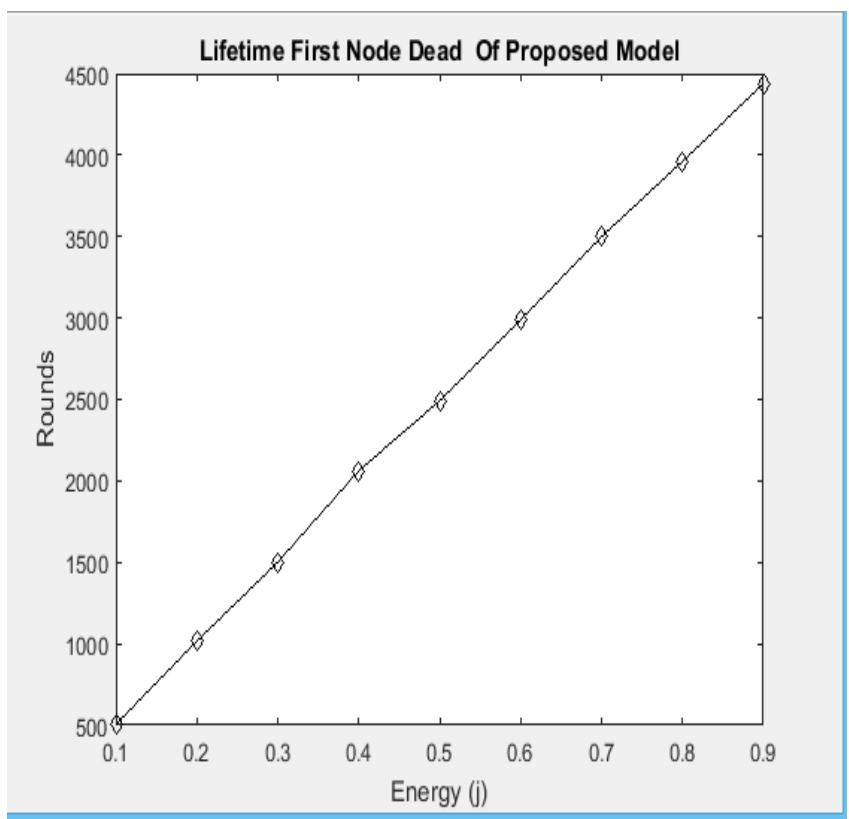

Figure 2: Lifetime of the First node dead of the proposed model

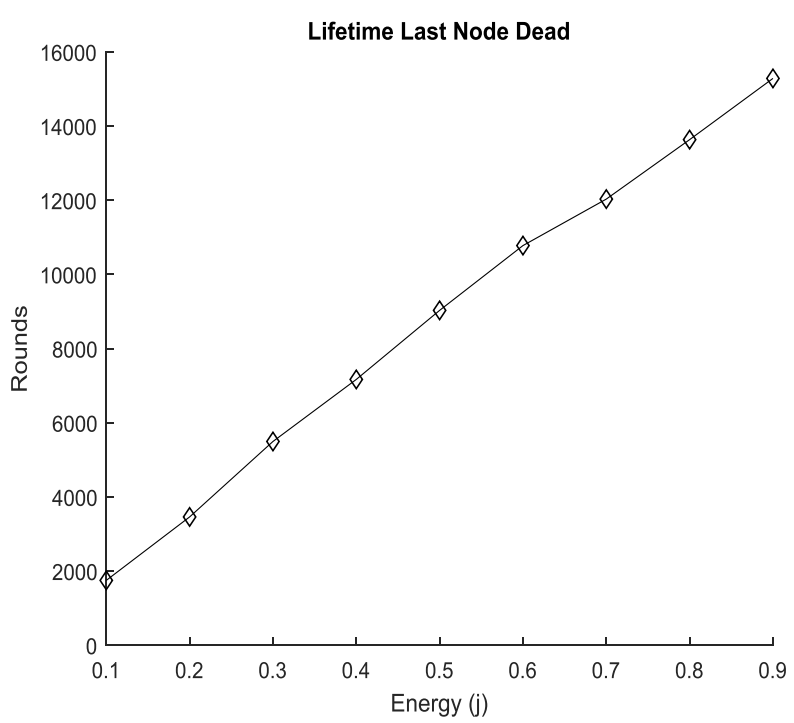

Figure 3: Last node dead with respect to the different energy level

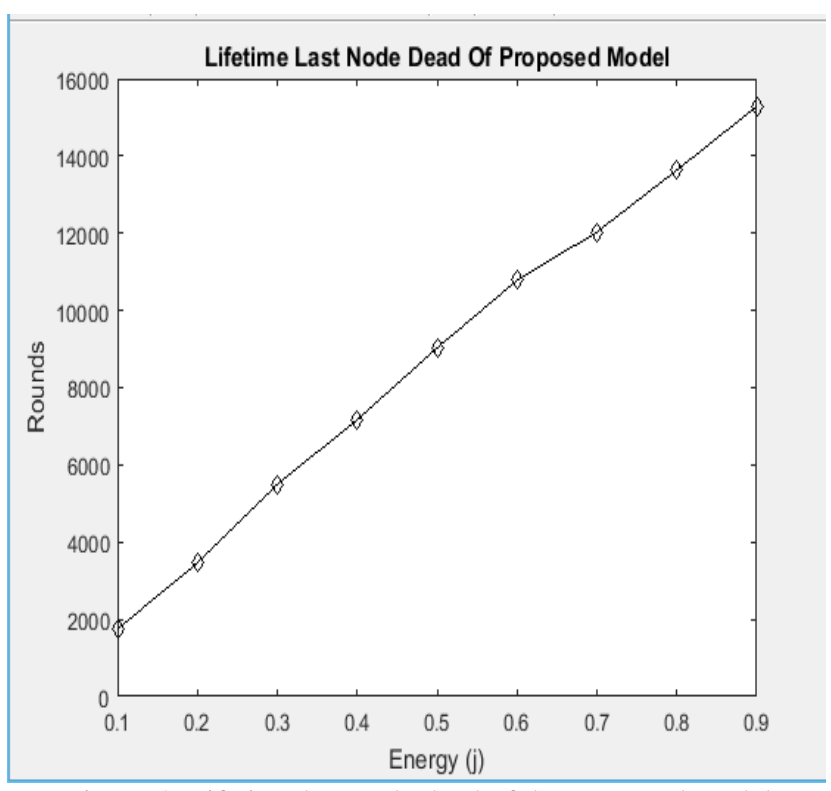

Figure 4: Lifetime last node dead of the Proposed model

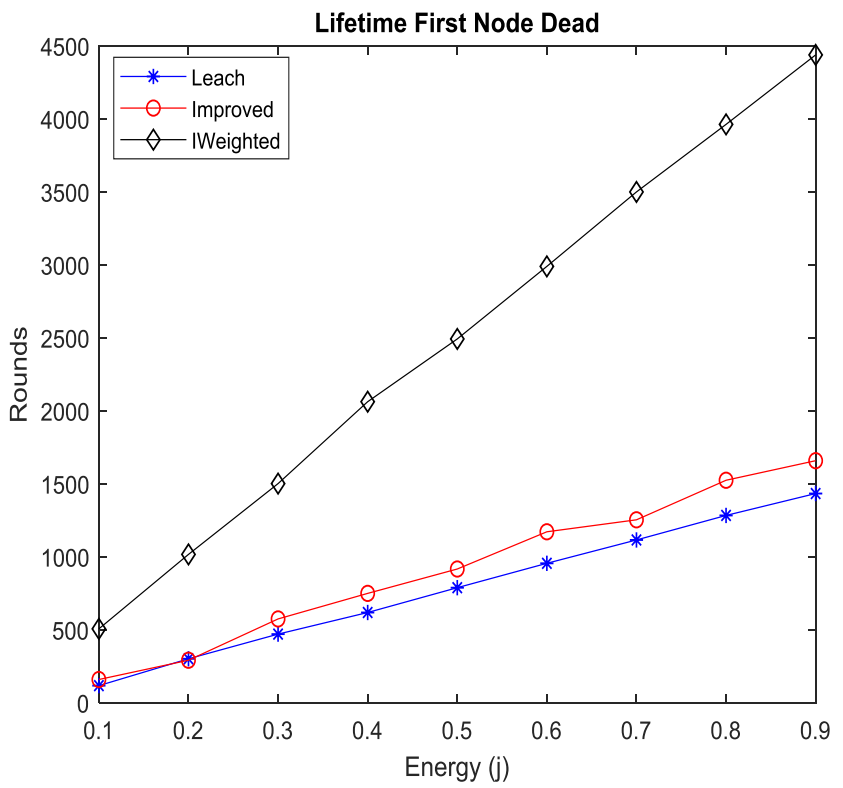

Figure 5: Comparison anlaysis of network lifetime in terms of first node dead 


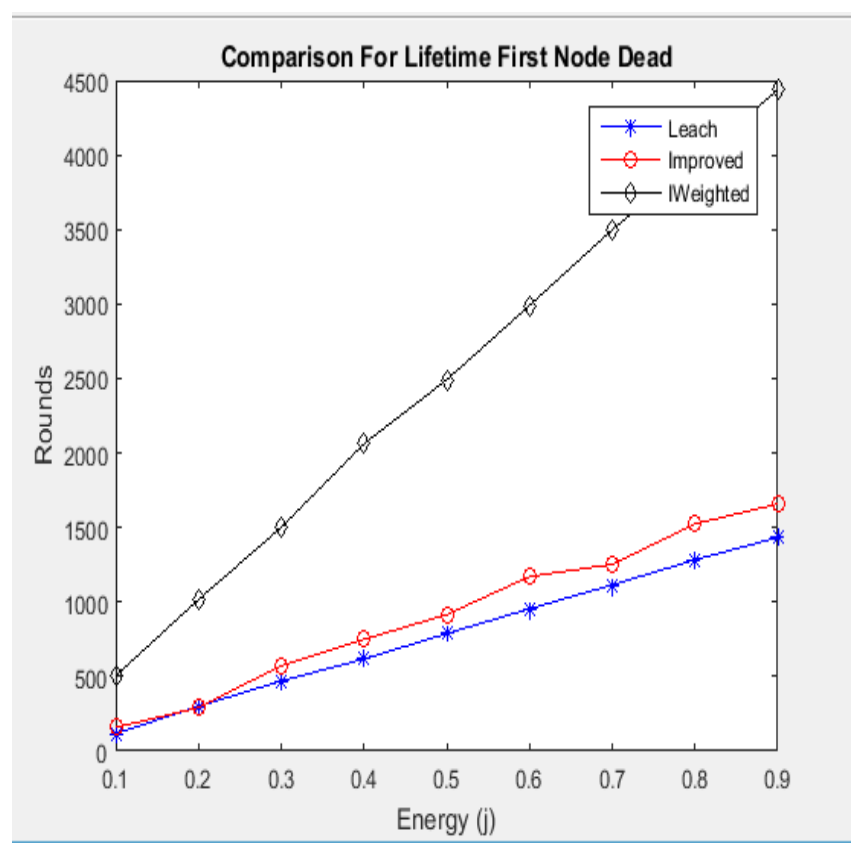

Figure 6:Comparison for lifetime first node dead

Table 3. Proposed network lifetime in terms of the first node dead

\begin{tabular}{|l|l|l|l|}
\hline Energy & LEACH & $\begin{array}{l}\text { Improved } \\
\text { LEACH }\end{array}$ & I-Weighted \\
\hline 0.1 & 117 & 159 & 506 \\
\hline 0.2 & 301 & 291 & 1016 \\
\hline 0.3 & 470 & 574 & 1501 \\
\hline 0.4 & 618 & 749 & 2062 \\
\hline 0.5 & 788 & 916 & 2492 \\
\hline 0.6 & 955 & 1171 & 2989 \\
\hline 0.7 & 1115 & 1253 & 3499 \\
\hline 0.8 & 1283 & 1524 & 3962 \\
\hline 0.9 & 1433 & 1658 & 4438 \\
\hline
\end{tabular}

The comparison analysis of proposed work with LEACH and Improved LEACH is shown in the graph. The comparison is done for network lifetime. The graph explains that the network in high energy are more lifetime in comparison to the network with lower energy. As we observed from the graph, when the initial energy is set to 0.2 joule then the network lifetime of LEACH and IMPROVED LEACH is near by 200 rounds whereas in this work it raches to the 1100 rounds approximately. When the energy level is set to the highest i.e. 0.9 then the network lifetime of the Iweighted mechanism is higher i.e. it works till 4500 rounds whereas the LEACH and improved LEACH exhaust after 1500 rounds. The observed facts and figures are calibrated in 2.

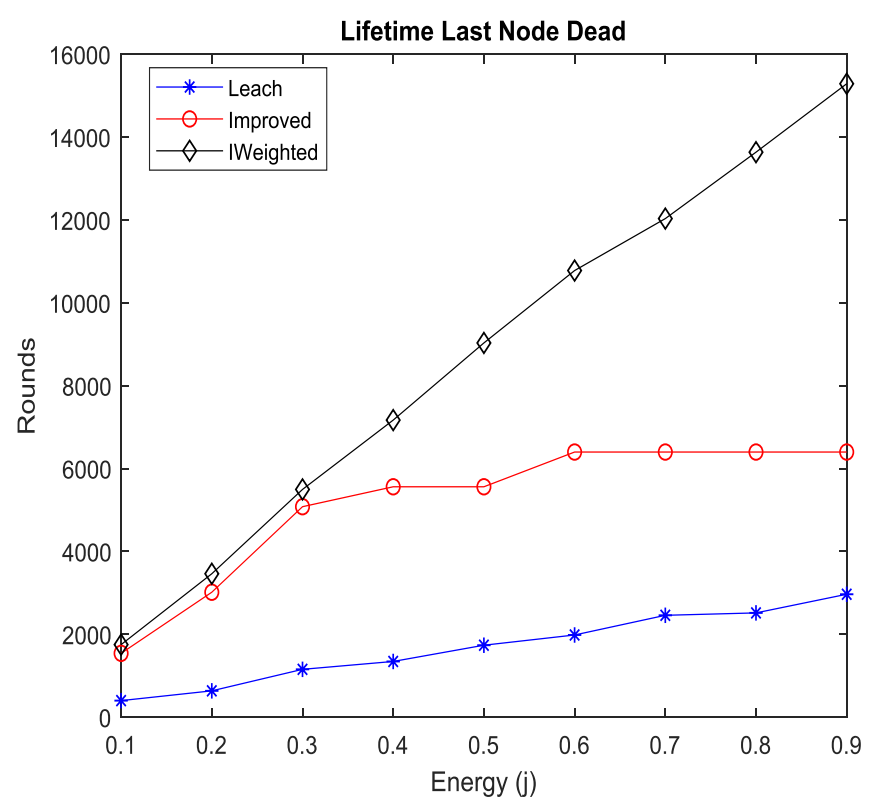

Figure 7: Comparison analysis of the network lifetime in terms of last node dead

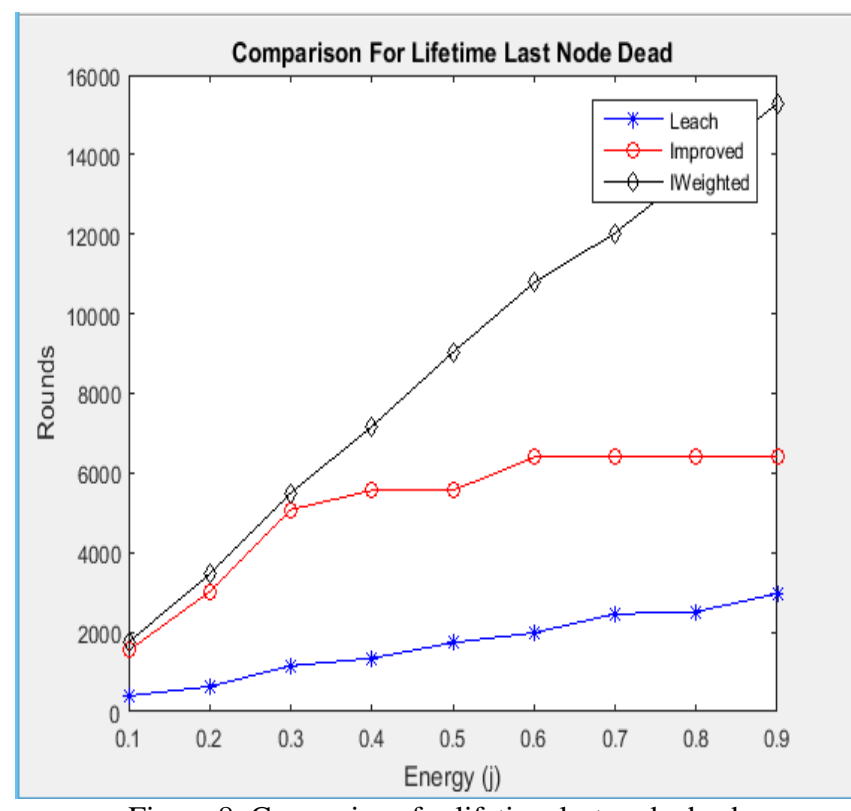

Figure 8: Comparison for lifetime last node dead

Table 4. Proposed network lifetime in terms of first node dead

\begin{tabular}{|l|l|l|l|}
\hline Energy & LEACH & $\begin{array}{l}\text { Improved } \\
\text { LEACH }\end{array}$ & I Weighted \\
\hline 0.1 & 399 & 1540 & 1750 \\
\hline 0.2 & 635 & 3015 & 3461 \\
\hline 0.3 & 1154 & 5079 & 5496 \\
\hline 0.4 & 1344 & 5560 & 7169 \\
\hline 0.5 & 1736 & 5560 & 9031 \\
\hline 0.6 & 1983 & 6398 & 10778 \\
\hline 0.7 & 2458 & 6398 & 12032 \\
\hline 0.8 & 2516 & 6398 & 13635 \\
\hline 0.9 & 2966 & 6398 & 15286 \\
\hline
\end{tabular}

Likewise, the graph in figure 7 elucidates the comparison of the network lifetime with respect to the last dead nodes in the network. The graph elucidates that the last node dead in Iweighted technique is found at 2000 rounds with the lower rate of energy at the nodes i.e. 0.2. When the 
amount of energy is increases to the 0.5 then the last node dead is located at 8200 rounds approximately. The similarly at the reach of 0.9 joule of energy the last dead node is located at the completion of 15000 rounds. Whereas for traditional schemes, the highest network lifetime is recorded till 2000 and 6000 rounds of communication respectively. The observations of the graph are shown in 4 .

\section{CONCLUSION}

WSN has an imperative role in a variety of domains where the fetching of data from the surrounding environment is the major task. The simulation of this work is done on the basis of the different amount of initial energies of the sensors with respect to the first node dead and the last node dead. The results conclude that the system produced is quite easy to access, as there is no constraint of limited range. The comparison is done with the traditional LEACH and IMPROVED LEACH protocol and we concluded that the resultant I weighted protocol is better and effective in terms of energy and network lifetime.

\section{REFERENCES}

[1] Shunmin Wang, "Application of high precision accuracy irrigation based on the fuzzy spatial data mining in 4G", IEEE, International Conference on Intelligent Human-Machine Systems and Cybernetics, 2014. Sandeep Kaur, Deepali, "An automatic irrigation system for different crops with WSN”, IEEE,2017 pp 407-411.

[2] Jin Wei and Gihan J. Mendis, "A Deep Learning-Based Cyber-Physical Strategy to Mitigate False Data Injection Attack in Smart Grids", IEEE, 2016.

[3] Peng Zhang, Qian Zhang, Fusheng Liu, Changqing Song, "The Construction of the Integration of Water and Fertilizer Smart Water Saving Irrigation System Based on Big Data", IEEE, International Conference on Computational Science and Engineering, 2017.

[4] Sandeep Kaur, Deepali, "An automatic irrigation system for different crops with WSN", IEEE, pp 407-411, 2017.

[5] Manpreet Kaur, Amarvir Singh, "Detection and Mitigation of Sinkhole Attack in wireless sensor network", IEEE, International Conference on Micro-Electronics and Telecommunication Engineering, 2016.

[6] Ravi Kishore Kodali , Borade Samar Sarjerao, "A Low Cost Smart Irrigation System Using MQTT Protocol”, IEEE, 2017.

[7] Mahammad Shareef Mekala, Dr P. Viswanathan, "A Novel Technology for Smart Agriculture Based on IoT with Cloud Computing", IEEE, 2017.

\section{AUTHORS PROFILE}

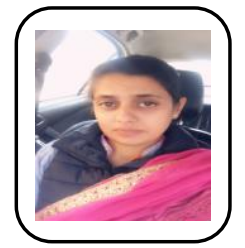

Miss Parneet Kaur received her degree in B.Tech in ECE from PTU Jalandhar in the 2015. She is currently pursuing M.Tech in ECE from PTU Jalandhar. Her research interest are in the field of wireless communication and Networking.

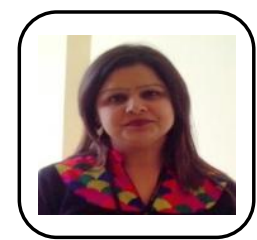

Dr. Pooja Sahni is working as Professor in Chandigarh Engineering College, Mohali, India. She has more than 15 years of experience, many 11 national and 16 international journal research papers to his credit and filed five patents. Her area of interest are Wireless Communication.

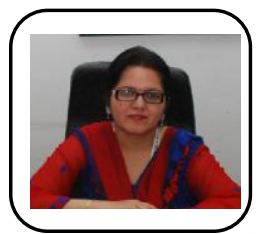

Dr. Sukhdeep Kaur is working as Professor in Chandigarh Engineering College, Mohali, India. She has more than 18 years of experience, many 15 national and 20 international journal research papers to his credit. Her areas of interest are Wireless Communication and Antenna design. 